\title{
Nonlinear Integration of Visual and Haltere Inputs in Fly Neck Motor Neurons
}

\author{
Stephen J. Huston ${ }^{1,2}$ and Holger G. Krapp ${ }^{1,3}$ \\ ${ }^{1}$ Department of Zoology, University of Cambridge, Cambridge CB2 3EJ, United Kingdom, 22Division of Biology, California Institute of Technology, Pasadena, \\ California 91125, and ${ }^{3}$ Department of Bioengineering, Imperial College London, London SW7 2AZ, United Kingdom
}

\begin{abstract}
Animals use information from multiple sensory organs to generate appropriate behavior. Exactly how these different sensory inputs are fused at the motor system is not well understood. Here we study how fly neck motor neurons integrate information from two well characterized sensory systems: visual information from the compound eye and gyroscopic information from the mechanosensory halteres. Extracellular recordings reveal that a subpopulation of neck motor neurons display "gating-like" behavior: they do not fire action potentials in response to visual stimuli alone but will do so if the halteres are coactivated. Intracellular recordings show that these motor neurons receive small, sustained subthreshold visual inputs in addition to larger inputs that are phase locked to haltere movements. Our results suggest that the nonlinear gating-like effect results from summation of these two inputs with the action potential threshold providing the nonlinearity. As a result of this summation, the sustained visual depolarization is transformed into a temporally structured train of action potentials synchronized to the haltere beating movements. This simple mechanism efficiently fuses two different sensory signals and may also explain the context-dependent effects of visual inputs on fly behavior.
\end{abstract}

\section{Introduction}

Animals often combine information from multiple sensory organs to provide a more robust estimate of a behaviorally relevant variable. The neural signals generated by different sensory systems are diverse in structure and bandwidth which makes it challenging to understand how they are fused. One experimentally amenable example of such sensor fusion is the gaze stabilization pathway of the fly. Like in vertebrates, in which neurons integrate both visual and vestibular inputs to estimate self-motion ( $\mathrm{Gu}$ et al., 2008), flies monitor self-motions using several visual and mechanosensory systems (Hengstenberg, 1991). A subset of visual interneurons, the horizontal and vertical system (HS and VS) lobula plate tangential cells (LPTCs) respond to panoramic retinal image shifts or "optic flow fields" generated during rotations of the fly about different body axes (Hausen, 1993; Krapp, 2000; Egelhaaf et al., 2002; Taylor and Krapp, 2007; Elyada et al., 2009). These LPTCs project directly, and also indirectly via descending neurons, to neck motor neurons (NMNs) responsible for gazestabilizing head movements (Strausfeld et al., 1987; Strausfeld and Gronenberg, 1990). Fast self-rotations are also detected by the halteres (Fig. 1a). Halteres are mechanosensory organs of equilibrium, functionally equivalent to the vertebrate vestibular

Received June 19, 2009; revised Aug. 21, 2009; accepted Aug. 29, 2009.

S.J.H. was supported by a Medical Research Council Ph.D. Studentship. H.G.K. was supported by the Royal Society and by European Office of Aerospace Research and Development Grant FW8655-05-1-3066. We thank J. Niven, T Matheson, S. Laughlin, K. Longden, and M. Parsons for helpful discussions. We are especially thankful to S. Rogers for providing help both with statistics and with setting up the intracellular electrophysiology. Two anonymous referees provided suggestions that improved this manuscript.

Correspondence should be addressed to either of the following: Stephen J. Huston, Division of Biology, California Institute of Technology, Pasadena, CA 91125, E-mail: Huston@caltech.edu; or Holger G. Krapp, Department of Bioengineering, Imperial College London, London SW7 2AZ, UK, E-mail: h.g.krapp@imperial.ac.uk.

DOI:10.1523/JNEUROSCI.2915-09.2009

Copyright $\odot 2009$ Society for Neuroscience ～0270-6474/09/2913097-09\$15.00/0 system. They consist of club-like anatomical structures that beat up and down at the same frequency, but antiphase, to the wings and detect the Coriolis forces imposed upon them by rotations of the fly (Pringle, 1948; Nalbach, 1994; Nalbach and Hengstenberg, 1994; Dickinson, 1999; Fox and Daniel, 2008). In addition to measuring self-rotations, the halteres also have sensory hair fields that can monitor their own oscillatory motion during straight flight (Nalbach, 1994; Dickinson, 1999).

Both the visual system and the halteres detect self-rotations of the fly, but in very different ways. The VS and HS cells of the visual system are more sensitive to comparatively slow rotations of the fly, whereas the halteres are more sensitive to faster rotations (Hengstenberg, 1991; Sherman and Dickinson, 2003). The haltere primary sensory neurons fire in phase with the fast haltere oscillations (Pringle, 1948; Fox and Daniel, 2008) whereas, over short time intervals, VS and HS cells respond to self-rotation with a sustained change in membrane potential (Hausen, 1982; Hengstenberg, 1982).

How is information from both the fast, oscillatory haltere system and the slower, nonoscillatory visual system integrated in the gaze stabilization pathway? To answer this question we recorded from NMNs that integrate both visual LPTC and haltere inputs (Sandeman and Markl, 1980; Strausfeld and Seyan, 1985; Milde et al., 1987; Huston and Krapp, 2008) to control compensatory head movements (Hengstenberg, 1991). We found that, in some NMNs, visual stimuli will only produce action potentials in the presence of a simultaneous haltere input. In addition, we describe and analyze the subthreshold events underlying this "gating-like" effect.

\section{Materials and Methods}

Electrophysiology

All experiments were performed on female, 1- to 3-d-old blowflies (Calliphora vicina) from the Cambridge Department of Zoology colony. The fly's legs and wings were removed and it was mounted ventral side up 

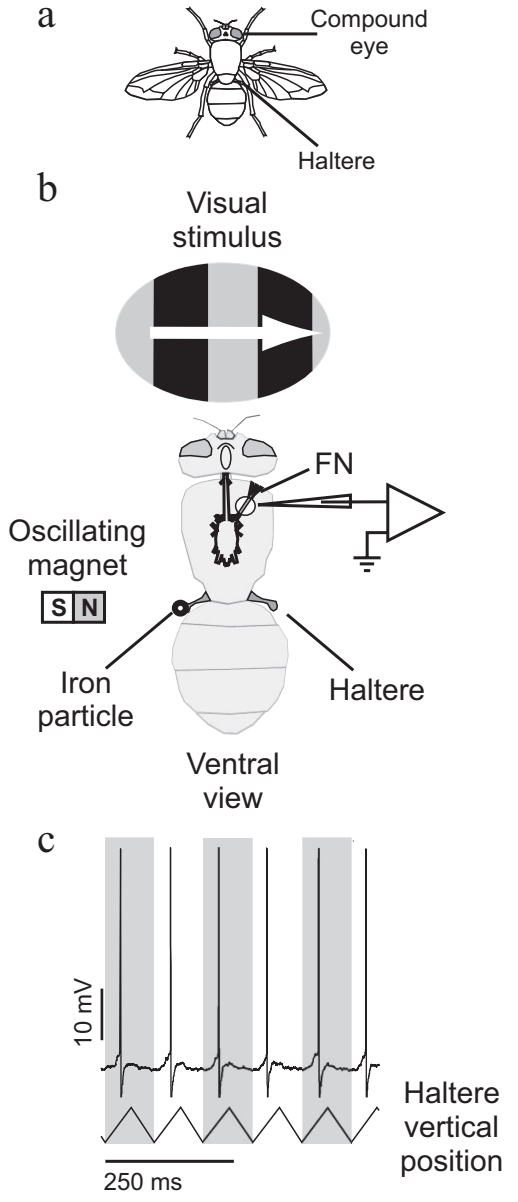

Figure 1. Experimental configuration. $\boldsymbol{a}$, Diagram of a blowfly showing the anatomical location of the sensory organs stimulated in this study. $\boldsymbol{b}$, Intracellular recordings were made from the FN axons through a small hole cut in the cuticle of the fly's ventral side. The movement of one of the halteres was controlled magnetically by attaching a small iron particle (shown larger than actual size) to the haltere and then vertically oscillating a magnet close to the haltere. Visual stimuli were presented on a CRT in the fly's frontal visual field. c, A sample trace of a right FN NMN intracellular response to oscillating the contralateral haltere through its stroke plain. The stimulus elicited action potentials phase locked to the haltere oscillation cycle. Calibration, $250 \mathrm{~ms}$.

upon a custom made holder. The ocelli were obscured using black paint. The head was aligned with the visual stimulus using the pseudopupil (Franceschini, 1975) and fixed with beeswax. The neck sclerites were waxed to reduce movement. A small window was cut in the cuticle and the air sacs teased aside, exposing the fly's frontal nerve (FN) and the extreme anterior portion of the prothoracic ganglion (Fig. 1b). Large neck muscles not innervated by the FN were cut to reduce movement. For the intracellular recordings, a $0.025-\mathrm{mm}$-diameter silver wire hook was placed under the FN to add support and act as an indifferent electrode. To further reduce movement, fine cactus spines were used to support the frontal nerve root and anterior portion of the prothoracic ganglion; insect pins were not used to avoid any interaction with the haltere stimulus' magnetic field. The preparation was kept moist with fly Ringer solution (Hausen, 1982). Thick-walled borosilicate glass micropipettes (resistance 70-120 M $\Omega$ ) filled with $2 \mathrm{M}$ potassium acetate were used to record from frontal nerve motor neuron axons within either the left or right $\mathrm{FN}$ at a distance of $\sim 0.3 \mathrm{~mm}$ along the nerve from the prothoracic ganglion. A recording was accepted only if the recorded resting membrane potential was stable and within the range from -55 to $-75 \mathrm{mV}$. Stable recordings lasted for 10-60 min. Occasionally the NMN would fire a burst of action potentials (supplemental Fig. 1, available at www.jneurosci.org as supplemental material); these bursts were correlated with haltere beating and contraction of the leg muscles and have been described previously (Sandeman and Markl, 1980; Milde et al., 1987). Any data taken during these bursts were discarded and the experimental trial was repeated. The neural signals were amplified 10-fold, low-pass filtered at $6 \mathrm{kHz}$ by an NPI SEC-10L amplifier operating in bridge balance mode, and then amplified 5 -fold by a custom-made direct-current amplifier. Data were acquired at $20 \mathrm{kHz}$ through a $\mathrm{Na}-$ tional Instruments PCI-6025E board on a computer running Matlab (Mathworks). The methodology for the extracellular recordings was similar except that we recorded from the other neck nerves in addition to the FN. Differential hook electrode recordings were made from the NMNs at their neck muscle arborizations; see the study by Huston and Krapp (2008) for more details.

\section{Stimulus generation and presentation}

Visual stimuli. Visual stimuli were presented on green cathode ray tube (CRT, P31 phosphor) driven by an Innisfree Picasso Image generator at a refresh rate of $182 \mathrm{~Hz}$. The CRT was mounted on a separate platform to the preparation in an attempt to mechanically isolate the two. A transparent electrical shield was placed in front of the CRT to reduce electrical noise. The CRT was placed directly in front of the fly (Fig. 1b) at a distance of $7.4 \mathrm{~cm}$ so that the circular screen aperture subtended a visual angle $62.6^{\circ}$ in diameter. Square wave gratings of $96 \%$ contrast and $10^{\circ}$ spatial wavelength were moved with a temporal frequency of $5 \mathrm{~Hz}$ in 1 of 16 different directions. Between grating presentations, the fly was shown a $6 \mathrm{~s}$ blank screen with the same mean luminance as the grating (18 $\mathrm{cd} / \mathrm{m} 2$ ) to allow for recovery from any possible adaptation. When the haltere stimulus was used in conjunction with the visual stimulus, the haltere stimulus was started $2 \mathrm{~s}$ before the visual stimulus. This delay ensured that the visual stimulus occurred well after the bursting activity that often accompanied haltere stimulus onset.

Haltere stimuli. While recording from a NMN, both halteres were touched in succession with a fine plastic tube. In all cases, touching one haltere would elicit many more action potentials than touching the other. This difference was clear-cut and enabled the unambiguous identification of the "preferred haltere" of the NMN, which was defined as the haltere whose movement would elicit the most action potentials. The haltere stimulator was aligned with the fly's left haltere. Thus, in the case of recordings from the left FN, the stimulated left haltere was the ipsilateral haltere, and in the case of right FN recordings, the contralateral haltere. By recording from left and right nerves in different experiments, recordings were obtained both when the haltere stimulus was on the preferred haltere and when the stimulus was on the "nonpreferred" haltere.

The calypter covering the haltere to be studied was removed. To control haltere movement in the extracellular experiments, a Ling Vibrator (model 101, Ling Dynamic Systems, Royston) was attached directly to the fly's haltere using solvent-free adhesive (Bostik). In the intracellular experiments the tip of the fly's left haltere was coated in solvent-free adhesive and iron powder $(<212 \mu \mathrm{m}$ particles, Sigma-Aldrich) and then moved magnetically via a $4 \times 3 \mathrm{~mm}$ neodymium magnet attached to a Ling Vibrator (Fig. 1b). The posterior portion of the eye on the same side as the stimulated haltere was painted black to block any visual input from the moving haltere stimulus. The haltere was held at an azimuth angle approximately equal to that of the resting haltere $\left(30^{\circ}\right.$ posterior from the mediolateral axis when viewed from above) and oscillated vertically with an amplitude of $\sim 50^{\circ}$. The haltere was oscillated vertically at frequencies ranging from $10.5-105 \mathrm{~Hz}$ in either a square-wave or triangular-wave pattern. A triangular waveform is closer to the fly's natural haltere beating movements than a square or sine wave (Nalbach, 1993; Fayyazuddin and Dickinson, 1996). Nonoscillating "ramp-and-hold" stimuli (Matheson and Ditz, 1991) were also applied to the haltere. In this case, the haltere was held in its lowest position for $200 \mathrm{~ms}$, raised at constant velocity to its highest position (equivalent to half a triangle wave, or "ramp"), and then held there for another $200 \mathrm{~ms}$. The speed of movement during the ramp transitions between the low and high positions was such that it was identical to the speed of movement in either the 10.5 $\mathrm{Hz}$ or $105 \mathrm{~Hz}$ triangle-waveform stimuli. At the end of each experiment the haltere stimulus was run while decoupled from the haltere to check 
a

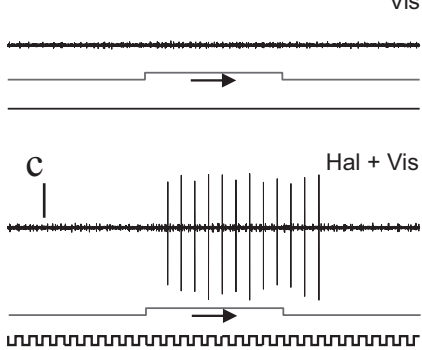

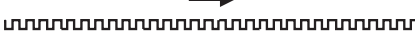

$1 \mathrm{~s}$ b

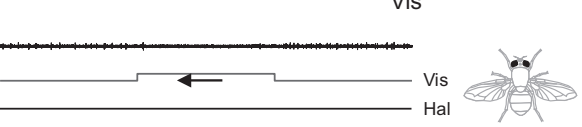

d $\quad$ Hal + Vis
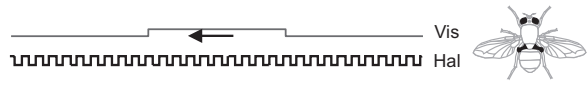

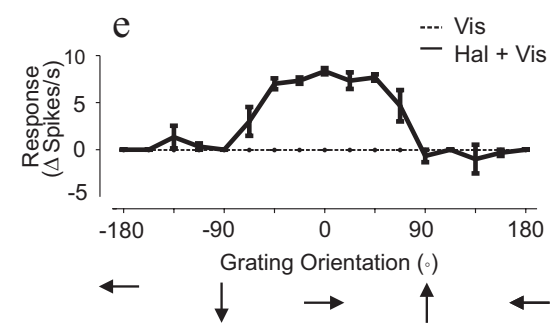

Figure 2. Extracellular responses of a right ventral cervical nerve NMN to combined ipsilateral haltere and visual stimulation. $\boldsymbol{a}$, $\boldsymbol{b}$, Extracellular responses to visual motion stimuli. The direction of visual motion is indicated by arrows. $\boldsymbol{c}, \boldsymbol{d}$, Responses to the same visual stimuli as in $\boldsymbol{a}$ and $\boldsymbol{b}$ shown in combination with ipsilateral haltere stimulation. The time course of the visual (Vis) and haltere (Hal) stimuli are shown below each extracellular trace. Vertical calibration, $0.25 \mathrm{mV}$; horizontal calibration, 1 s.e, Responses of the same neuron plotted against 16 directions of visual motion. Responses are shown to visual stimulation alone (dotted line) and to combined visual and haltere stimulation (solid line). $n=3$ for each grating orientation; error bars $=$ SES.

that no response to general vibration or magnetic artifact was present in our recordings.

\section{Data analysis}

Extracellular signals were spike sorted using template-matching software custom written in Matlab (Mathworks). For both the extracellular and intracellular experiments, the action potential response to visual motion was defined as the difference between the number of action potentials during a $1 \mathrm{~s}$ grating presentation and the number of action potentials during the preceding $1 \mathrm{~s}$ in which only the blank screen was presented. The action potential response to visual stimuli during simultaneous haltere stimulation was similarly defined as the difference between the number of action potentials during $1 \mathrm{~s}$ of concurrent visual and haltere stimulation and the number of action potentials during the preceding $1 \mathrm{~s}$ time interval of just haltere stimulation. By comparing the responses of one unit to 16 different, equally spaced directions of visual motion, a directional tuning curve was constructed. The tuning curve peak was estimated by finding the phase of the first harmonic in a Fourier transformation of the tuning curve. The peak of the tuning curve gives the direction of visual motion to which the cell responds most strongly, referred to here as the cell's "preferred direction." In those intracellular experiments in which there were no action potentials in response to visual motion, the subthreshold response was defined as the difference between the mean membrane potential during $1 \mathrm{~s}$ of grating motion and the mean membrane potential during the preceding second while presenting the blank screen. Using this response, a tuning curve and estimate of the preferred direction were produced in the same manner as for the action potential responses. Membrane potential variance was calculated only using the second half of a visual response to ensure the response had reached steady state and avoid any onset transients. Some intracellular recordings were taken from the fly's right FN, whereas others were taken from the fly's left FN. To allow the comparison of these results, the directional tuning curves acquired from units in the right FN were mirror transformed horizontally over the vertical axis so as to be the same as those of the equivalent units in the left FN. Circular statistics were performed in custom written Matlab programs, and all noncircular statistics were performed in SPSS (SPSS).

The phases of postsynaptic potentials (PSPs) relative to the haltere stimulus were determined from the time at which the membrane poten-

tial increase was steepest. We estimated this time point by low-pass filtering the subthreshold waveform at $100 \mathrm{~Hz}$ with a linear phase filter and then finding the peak in the derivative of the filtered signal.

\section{Results}

To characterize how motor neurons integrate inputs from two different sensory organs, we recorded the extracellular responses of NMNs while presenting haltere and visual stimuli, both individually and in combination. Then, to reveal the subthreshold events underlying the NMN multisensory integration, we performed intracellular recordings from the NMNs of the FN.

The visual responses of NMNs have been studied previously (Milde et al., 1987; Huston and Krapp, 2008); it was shown that only a subset of NMNs produce action potentials in response to visual stimuli. For convenience we refer to those NMNs that respond to visual stimuli with action potentials as exhibiting "type I behavior" ["visual" NMNs in the terminology of Milde et al. (1987)] and those that do not as exhibiting "type II behavior" ["nonvisual" NMNs in the terminology of Milde et al. (1987)]. This terminology is not meant to imply that there are two distinct classes of NMN but instead is used for convenience to distinguish between NMN responses based upon just one particular criterion: the action potential response to visual stimuli. We obtained intracellular recordings from 10 type I FN NMNs and 27 type II FN NMNs; in addition, we also obtained extracellular recordings from 4 type I NMNs and 6 type II NMNs.

\section{Type II NMNs respond to visual stimuli only during haltere stimulation}

Response to haltere stimuli

We gently touched both of the halteres with a fine plastic tube. All NMNs fired action potentials when one of the halteres was touched. In all cases, the response to touching one haltere was much stronger than to touching the other; this effect was robust and unambiguous. We refer to the haltere that the neuron was most sensitive to as the preferred haltere. For some NMNs the preferred haltere was ipsilateral; for others, it was contralateral. The NMNs fired action potentials in response to both vertical and horizontal displacements of the preferred haltere.

Using a controlled haltere stimulus, we induced vertical oscillations in the preferred haltere; this resulted in action potentials phase locked to the stimulus (Fig. 1c). We measured how accurate this phase locking was by finding the haltere stimulus phase of the first action potential in each haltere stimulus cycle. For each cell we determined the smallest time window that, when centered on a cell's mean firing phase, could encompass $90 \%$ of these action potentials. The median window width was $3.6 \pm 7.9 \mathrm{~ms}$ (median \pm interquartile range, $n=12$ cells). Thus, $90 \%$ of these action potentials occurred within $4 \%$ of the haltere stimulus period. In 9 of those 10 experiments in which the stimulus was applied to the nonpreferred haltere, the stimulus did not elicit action potentials (although in intracellular experiments it did result in PSPs), regardless of the oscillation amplitude. All haltere 

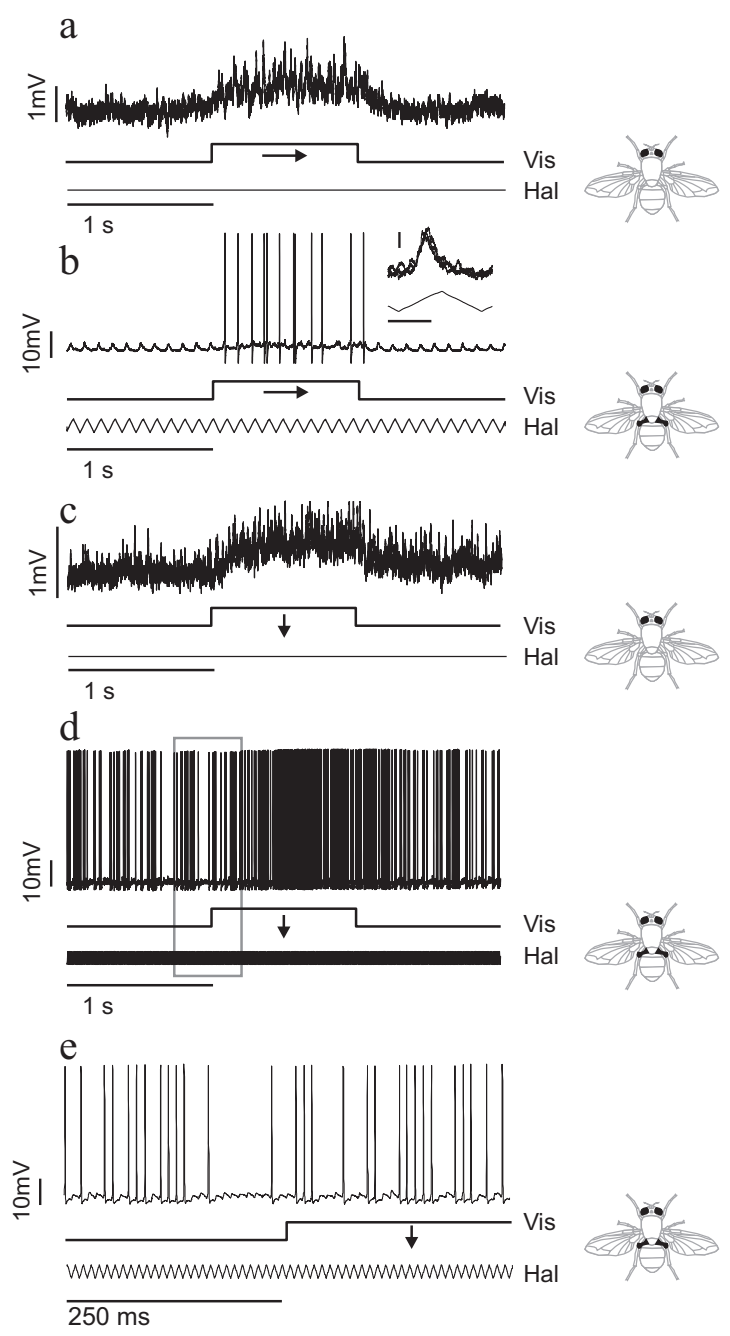

Figure 3. Intracellular responses of frontal nerve NMNs to visual and haltere stimuli. $\boldsymbol{a}-\boldsymbol{d}$, Intracellular responses of two different frontal nerve NMNs to visual and haltere stimuli. $\boldsymbol{a}, \boldsymbol{c}$, Small subthreshold depolarization resulting from visual stimuli moving in each NMN's preferred direction: horizontally left to right for $\boldsymbol{a}$ and vertically downward for $\boldsymbol{c} . \boldsymbol{b}, \boldsymbol{d}$, NMNs' responses to the same direction of visual stimuli when combined with concurrent haltere stimulation (note that the vertical scale bars in $\boldsymbol{a} / \mathbf{c}$ and $\boldsymbol{b} / \boldsymbol{d}$ are different). A haltere stimulation of $10.5 \mathrm{~Hz}$ was used in $\boldsymbol{b}$, and $105 \mathrm{~Hz}$ stimulation was used in $\boldsymbol{d}$. The inset in panel $\boldsymbol{b}$ shows an overlay of PSPs resulting from just haltere stimulation in the first second of the trial. Inset horizontal calibration, $50 \mathrm{~ms}$; inset vertical calibration, $1 \mathrm{mV}$. $\boldsymbol{e}$, The boxed data from $\boldsymbol{d}$ plotted on a finer timescale. Lines below the neural traces indicate the visual (Vis) and haltere (Hal) stimulus time courses.

stimulation data presented in this study were taken from experiments in which the haltere stimulus was applied to the preferred haltere.

Response to combined visual and haltere stimuli

When the halteres were not moving, type II NMNs did not produce action potentials in response to any of the directions of visual motion stimuli we presented (Fig. $2 a, b$ ). However, when the haltere stimulus was applied concurrently with visual stimuli, certain directions of visual motion could elicit additional action potentials from type II NMNs (Fig. 2c). In some NMNs, the neuron would not fire action potentials in response to the haltere stimulus alone but would in response to the combined visual and haltere stimulus (Fig. 2). Other NMNs would fire action potentials in response to the haltere stimulus alone and the addition of the visual stimulus could modulate this baseline rate of action a
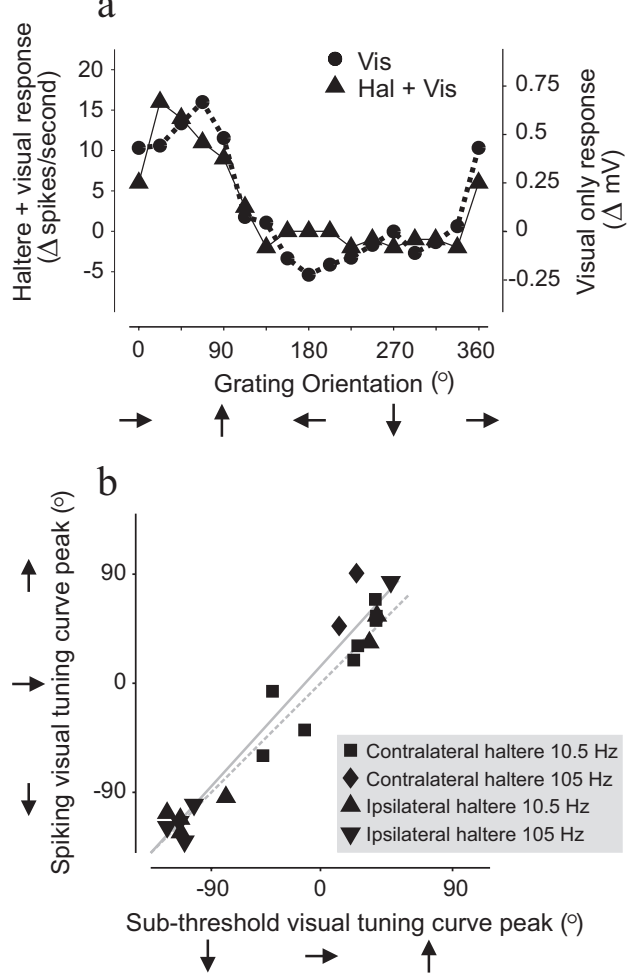

Figure 4. NMNs' subthreshold responses are tuned to the same directions of visual motion as their action potential responses. $\boldsymbol{a}$, Example of a visual directional tuning curve from a right FN NMN. The circular symbols and dashed line show the average amplitude of the NMN's subthreshold response to different directions of visual motion. The triangular symbols and solid line show the same NMN's action potential response to combined contralateral haltere oscillation and visual motion stimuli. The subthreshold and action potential tuning curves are very similar. $\boldsymbol{b}$, Correlation between subthreshold and action potential tuning curve peaks for all FN NMNs studied. Subthreshold visual tuning curve peaks are plotted against the peaks of the action potential tuning curves obtained during combined visual and haltere stimulation. Different symbols indicate the frequency of haltere stimulation and which haltere it was applied to in each individual experiment. The data points fall around a line of slope of $\approx 1$, suggesting that for all tested NMNs $(n=21)$ the subthreshold and action potential visual tunings were virtually identical. The solid gray line shows the result of a linear regression and the dotted gray line shows a line of unity slope and zero intercept.

potentials (Fig. 3d; supplemental Figs. 2 and 3, available at www. jneurosci.org as supplemental material).

The additional action potentials induced by a visual stimulus during haltere stimulation were compared across 16 different directions of visual motion. When the responses to the visual stimulus were plotted against direction of motion, they formed a single peaked tuning curve (Fig. $2 e$ ). In all six type II units recorded from extracellularly, the number of extra action potentials induced by a visual stimulus during haltere stimulation was significantly modulated by the direction of visual motion (visual tuning curves subjected to a nonlinear regression to a cosine, $p<$ 0.05 for all six units). In the majority of recordings the directional tuning was the result of stimulation in the preferred direction increasing the action potential rate. However, in at least one example the directional tuning was due to visual motion in the antipreferred direction reducing the action potential rate (supplemental Fig. 2, available at www.jneurosci.org as supplemental material), suggesting the possibility of an inhibitory visual input. By combining haltere and visual stimuli it was possible to map the visual receptive fields of those NMNs that do not normally respond to visual stimuli (supplemental Fig. 4, available at www. jneurosci.org as supplemental material). This was done in a 

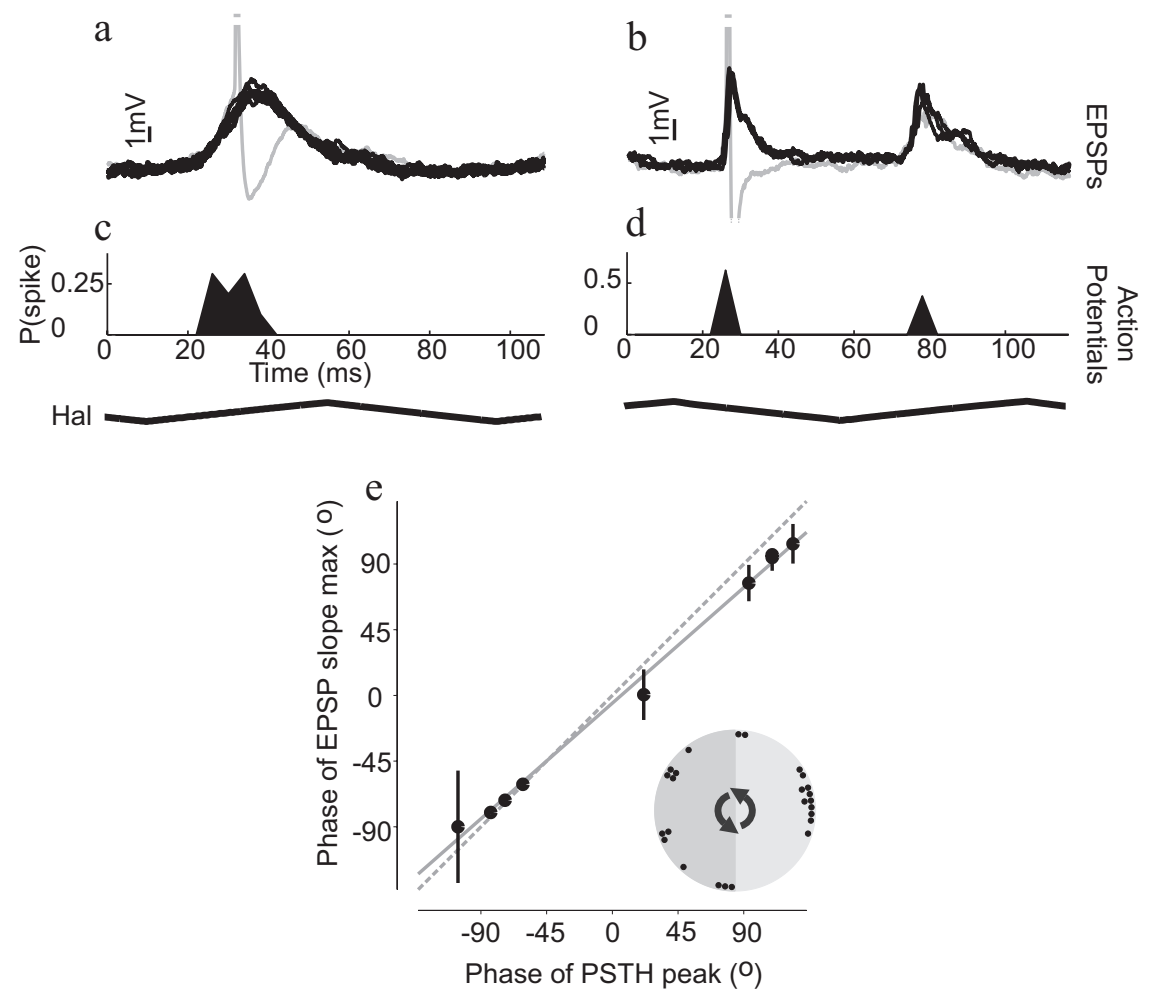

Figure 5. Visually induced action potentials and haltere-induced PSPs occur at the same phase of haltere movement. $\boldsymbol{a}, \boldsymbol{b}$, The compound PSPs occurring during one cycle of haltere-only stimulation are overlaid to show their phase locking to the haltere stimulus (haltere stimulus waveform shown below $\boldsymbol{c}$ and $\boldsymbol{d}$ ). Clipped action potentials are also shown in gray. $\boldsymbol{a}$ and $\boldsymbol{b}$ are taken from two different example neurons. $\boldsymbol{c}, \boldsymbol{d}$, Histograms giving the probability of action potentials occurring at different phases of the haltere stimulus cycle during combined visual and haltere stimulation. $\boldsymbol{c}$ and $\boldsymbol{d}$ are taken from the same neurons as $\boldsymbol{a}$ and $\boldsymbol{b}$, respectively, and were plotted on the same timescale; $4 \mathrm{~ms}$ wide bins were used to create the histograms. The compound PSP waveforms obtained during haltere-only stimulation occur at similar phases of the haltere stimulus waveform as the action potentials during combined stimulation. $\boldsymbol{e}$, The correlation between the timing of action potentials (during combined haltere and vision stimulation) and the timing of the PSPs' maximum slope (during just haltere stimulation). Both PSP and action potential times are plotted relative to the haltere stimulus phase. Each circle represents the mean phase for one neuron; $n=10$. Error bars are circular SDs. Some error bars are too small to be visible. The solid gray line is the result of a linear regression and the dotted gray line has unity slope and zero intercept. The circular inset in e shows the mean action potential phases for 25 cells that responded to contralateral haltere stimulation.

similar manner to previous studies of type I NMNs (Huston and Krapp, 2008).

Subthreshold visual and haltere inputs to type II NMNs

To elucidate the subthreshold mechanism underlying the nonlinear integration of haltere and visual inputs, we performed intracellular recordings from the axons of type II NMNs in the FN. As all recordings were taken from axons of the NMNs within the nerve itself (at least $200 \mu \mathrm{m}$ from the dendrites), all subthreshold events observed were small, but consistent and well above the noise level.

Visual stimulation in the cell's preferred direction resulted in a small, but significant, sustained depolarization of the membrane potential (Fig. $3 a$ ) (median depolarization $=0.5 \mathrm{mV}$, Wilcoxon signed-rank test, $p \ll 0.0001, n=27$ ). Consistent with an excitatory synaptic input, the variance of the membrane potential also increased during visual stimulation in the preferred direction (Fig. $3 a$ ) (median increase in SD of membrane potential $=0.04$ $\mathrm{mV}$, Wilcoxon signed-rank test, $p \ll 0.0001, n=27)$. The depolarization due to visual motion was highly dependent on the direction of visual motion, resulting in single peaked tuning curves (Fig. 4a). This directionality of the subthreshold visual response was statistically significant for all 27 type II units (visual tuning curves subjected to a nonlinear regression to a cosine, $p<0.05$ for all units). In all but 2 of the 27 type II units studied, the directional tuning was due to depolarization during motion in the units' preferred direction with only slight hyperpolarization in the units' antipreferred direction (Fig. 4a). The hyperpolarization during motion in the antipreferred direction was statistically significant, with a median value of $-0.1 \mathrm{mV}$ (Wilcoxon signed-rank test, $p \ll 0.0001, n=27)$. There was no statistically significant change in the membrane potential variance during antipreferred motion (median change in SD of membrane potential $=0.004 \mathrm{mV}$, Wilcoxon signed-rank test $p=0.26, n=27$ ).

Haltere stimulation resulted in compound PSPs and, in some cases, action potentials that were phase locked to the haltere stimulus waveform (Fig. 3b, inset). When we presented a visual stimulus in addition to the haltere stimulus, the NMN responded with additional phase-locked action potentials (Fig. $3 b$; supplemental Fig. 3, available at www.jneurosci.org as supplemental material).

Visual tunings of subthreshold and action potential responses are similar

For 21 type II units, we obtained visual directional tuning curves from both the subthreshold response during only visual stimulation and the suprathreshold action potential response during combined visual and haltere stimulation. For each unit, the two directional tuning curves were very similar (Fig. 4). The peaks of the subthreshold and suprathreshold tuning curves were strongly correlated (ranked nonparametric circular correlation, $\rho=$ 0.98, $p \ll 0.0001, n=21$ ) (Rao Jammalamadaka and Sengupta, 2001), falling upon a straight line with a slope close to 1 (slope of linear regression $=1.1$ ) (Fig. $4 b)$. This correlation between the visual tuning of the subthreshold and suprathreshold responses is consistent with the hypothesis that the subthreshold visual input is responsible for the visual modulation of the action potential output seen during combined haltere and visual stimulation.

Timings of subthreshold and action potential responses are similar The compound PSPs elicited by the haltere stimulus occurred at discrete points within the haltere oscillation cycle (Fig. $5 a, b$ ). In most NMNs only one compound PSP occurred per haltere stimulus cycle (Fig. 5a); however, in some NMNs the PSPs occurred at two different phases of the haltere stimulus (Fig. 5b). The action potentials induced by combined visual and haltere stimulation occurred at a similar phase to the compound PSPs elicited by haltere stimulation alone (Fig. $5 c, d$ ). This is true for all NMNs studied; when the phases of the PSPs and the action potential phases are plotted against each other they fall upon a straight line with a slope close to 1 (Fig. 5e) (circular correlation, $\rho=0.99, p=$ $0.002, n=10$, slope of linear regression $=0.9$ ). More NMNs responded during the midpoint of the haltere upstroke than at 
any other phase, but additional NMNs fired at many other phases (Fig. 5e, inset).

Although type I NMNs produce action potentials in response to visual stimuli alone, we found that simultaneous haltere stimulation could entrain the visually induced action potentials so they became phase locked to the haltere stimulus oscillation (supplemental Fig. 5, available at www.jneurosci.org as supplemental material). Haltere stimulation did not change type I NMNs' preferred direction of visual motion (supplemental Fig. 5, available at www.jneurosci.org as supplemental material), although in some units it did increase the response gain (data not shown). In the flight steering motor neuron MNB1, haltere and nonhaltere stimuli have been found to interact, shifting the phase of action potentials with functionally important consequences (Fayyazuddin and Dickinson, 1996, 1999). We tested whether visual stimuli induced any similar phase change in the NMN action potentials. No consistent action potential phase shift with the addition of visual stimuli was seen across the sampled NMN population (mean change in action potential phase with a visual stimulus in preferred direction: $-0.2 \pm 4.0 \mathrm{~ms} \mathrm{SD}, n=18$ ). However, such visually induced phase shifting was observed in a minority of units (data not shown).

\section{Effects persist at naturalistic haltere beating frequencies}

The majority of experiments described so far have used a haltere stimulus that oscillated vertically at a frequency of $10.5 \mathrm{~Hz}, \sim 10$ times slower than the frequency at which female blowflies beat their halteres during flight $(105-120 \mathrm{~Hz})$ (Pringle, 1948). We performed additional experiments using a haltere stimulus oscillating at a frequency of $105 \mathrm{~Hz}$ to determine if the effects observed at lower frequencies are still present with haltere oscillations in the frequency range seen during flight. These experiments showed that all the effects seen with a $10.5 \mathrm{~Hz}$ haltere stimulus were also present with a $105 \mathrm{~Hz}$ haltere stimulus. As with the slower stimulus, $105 \mathrm{~Hz}$ haltere stimulation resulted in phase-locked compound PSPs and action potentials in the NMNs (Fig. 6a,b), and the rate of these action potentials could be modulated with visual stimuli (Fig. 3d,e).

As before, we measured the phase of the first action potential occurring within each haltere stimulus cycle. For each cell we determined how large a time window was needed to encompass $90 \%$ of these action potentials. The median window width was $2.1 \pm 1.1 \mathrm{~ms}$ (median \pm interquartile range, $n=6$ cells). Thus, $90 \%$ of these action potentials occurred within $22 \%$ of the haltere stimulus period. To compare the action potential timing precision to that of the haltere sensory neurons, we measured the maximum vector strength and jitter of the action potential phases in the same way as previously obtained for haltere primary afferents (Fox and Daniel, 2008). The maximum vector strength $(0=$ no phase locking, 1 = perfect phase locking) for $105 \mathrm{~Hz}$ stimulation was 0.97 (Rayleigh test for nonuniformity, $p \ll 0.0001$ ), close to the value of 0.99 found for the haltere primary afferents (Fox and Daniel, 2008). The motor neurons had an average $0.6 \mathrm{~ms}$ jitter (measured as the SD in action potential timing), larger than the $0.2 \mathrm{~ms}$ value found for the primary afferents but still submillisecond (Fox and Daniel, 2008).

We investigated how the NMN responses could remain phase locked to the haltere stimulus even though the compound PSPs elicited by the $10.5 \mathrm{~Hz}$ stimulus were wide enough to summate if elicited at 10 times the frequency (Fig. $6 a$ ). To separate the velocity of haltere motion from the stimulus oscillation frequency, we subjected the halteres to ramp-and-hold stimuli. We moved the haltere of interest between a low and high position at two differ-

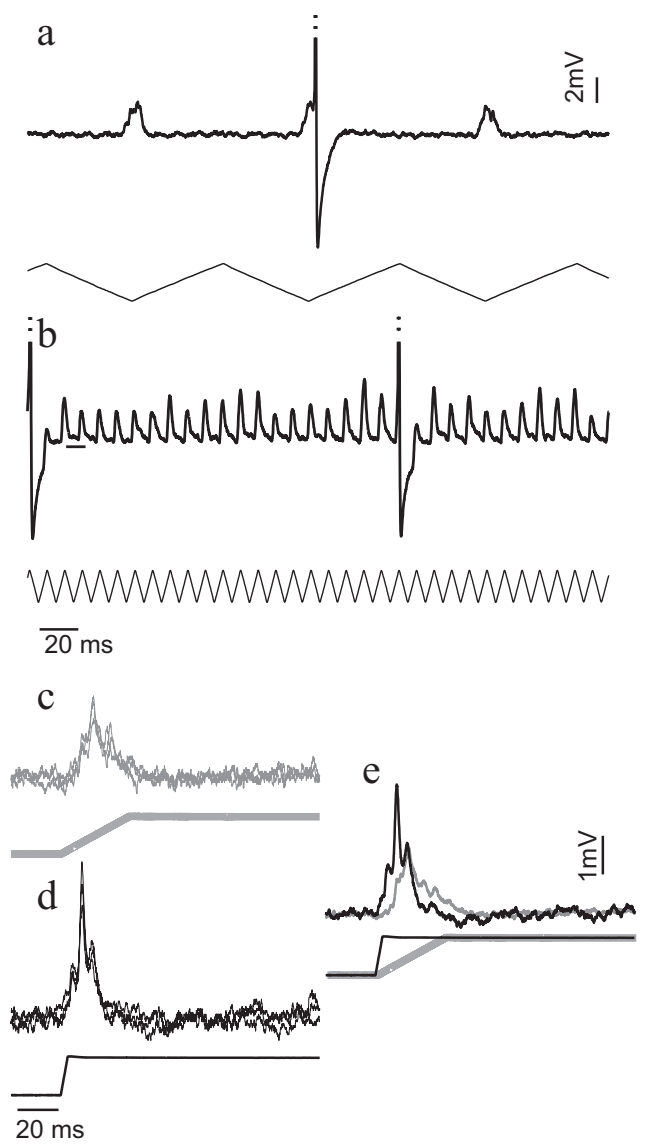

Figure 6. Phase locking persists at naturalistic haltere beating frequencies. $\boldsymbol{a}, 0$ ne FN NMN's response to haltere stimulation at a frequency of $10.5 \mathrm{~Hz}$. $\boldsymbol{b}$, The same NMN's response to $105 \mathrm{~Hz}$ haltere stimulation. The small horizontal line gives the resting potential of the neuron. Note that in both cases the PSPs and action potentials are phase locked to the stimulus, even though the compound PSPs occurring at $10.5 \mathrm{~Hz}$ are wide enough to summate if they were elicited at $10 \times$ the stimulation frequency. Action potentials have been clipped for display purposes. $c-e$, A different FN NMN's response to nonoscillatory ramp-and-hold stimuli in which the haltere was transiently moved from a low to high position at a speed equivalent to that occurring during a $10.5 \mathrm{~Hz}$ oscillation (c) or a $105 \mathrm{~Hz}$ oscillation $(\boldsymbol{d})$. $\boldsymbol{e}$, Averages of the waveforms in $\mathbf{c}$ and $\boldsymbol{d}$ : the gray line results from the slow haltere movement in c and the black line from the fast movement in $\boldsymbol{d}$. A sharpening of the compound PSP is evident during faster movements of the haltere.

ent velocities. The two velocities of haltere motion were chosen to match the haltere velocities seen during either the $10.5 \mathrm{~Hz}$ or the $105 \mathrm{~Hz}$ haltere oscillatory stimulus. When we moved the haltere at the slow speed the NMN produced a compound PSP (Fig. 6c,e) that was wide enough to summate and produce a tonic response if repeatedly elicited at $105 \mathrm{~Hz}$. However, when we moved the haltere at the speed experienced during $105 \mathrm{~Hz}$ stimulation, an additional sharp component was added to the compound PSP (Fig. $6 d, e$ ), possibly accounting for the response remaining phase locked at higher oscillation frequencies.

\section{Discussion}

To investigate how motor systems integrate inputs from different sense organs, we studied the responses of fly neck motor neurons to haltere and visual inputs both individually and in combination. Extracellular and intracellular recordings revealed that some NMNs do not produce action potentials in response to visual stimuli alone, but the same NMNs will produce action potentials in response to visual stimuli if the halteres are concurrently beating. Our evidence suggests that this gating-like effect is due to a small, 


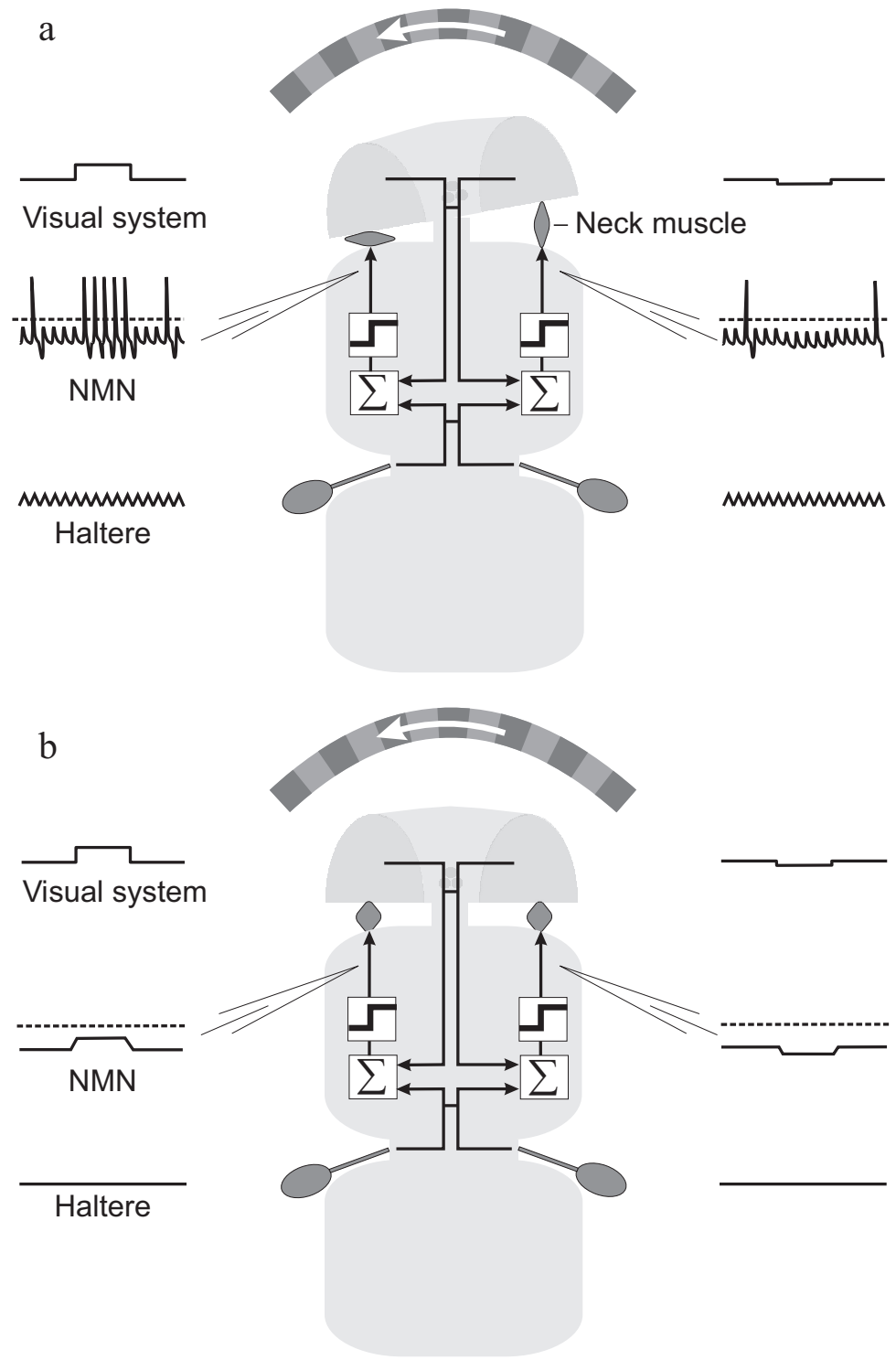

Figure 7. Nonlinear multisensory integration at motor neurons may explain behavioral results. A simple schematic illustrates the agreement between our electrophysiological results and previous behavioral findings. Visually induced head movements are seen only during flight and walking, not when the fly is at rest (Hengstenberg, 1991). $\boldsymbol{a}$, During flight or walking blowflies move their halteres (Sandeman and Markl, 1980). Visual inputs summate with those from the halteres and cross the action potential threshold (dotted line in NMN trace). This results in haltere phase-locked action potentials which activate neck muscles and initiate compensatory head movements. $\boldsymbol{b}$. When the fly is at rest, its halteres do not move. Our results suggest that under these conditions, the visual inputs to type II NMNs are subthreshold and thus cannot affect neck muscle activity, resulting in reduced head movements.

subthreshold visual input modulating the probability that haltereinduced PSPs become suprathreshold action potentials.

The action potential nonlinearity results in a gating-like effect The term "gating" is used in different ways by different authors. At the level of neurons, the term is either used to refer to a class of mechanisms that determine whether or not a synaptic input is effective (Katz, 2003) or as a phenomenological description of the way a neuron's action potential output behaves (Reichert et al., 1985; Reichert and Rowell, 1986). Here we use the term gating in the phenomenological sense of Reichert et al. (1985). We define gating as one sensory input influencing the action potential output of a neuron only if a certain condition is met, such as the presence of a specific motor pattern or a different sensory input (Reichert et al., 1985; Sillar and Roberts, 1988; Staudacher and Schildberger, 1998). In our experiments the responses of type II
NMNs meet this definition of gating in that visual inputs could affect the neuron's action potential rate, and thus the muscle activity, only if the haltere input was also present. In other words, the visual input is permissively gated by the haltere input as was previously hypothesized to occur in the NMNs for visual (Sandeman, 1980) and prosternal organ (Gilbert and Bauer, 1998) inputs. Conversely, the results can be equally well thought of as a visual modulation of the NMNs' responses to the haltere input, as the haltere-driven action potentials may provide necessary tonic muscle tension, which is then modulated by visual input.

The results of our intracellular recordings suggest that the gating-like effect is mediated by the threshold nonlinearity inherent to the generation of action potentials. We hypothesize that the descending visual inputs to $\mathrm{FN}$ type II NMNs (Haag et al., 2007; Wertz et al., 2008) are too small to generate action potentials on their own but can affect the probability that the haltere-induced PSPs become suprathreshold. Thus, the visual input can affect the neuron's action potential output only in the presence of another input, such as that from the halteres (Fig. 7). This simple mechanism suggests a way in which two very different sensory inputs may be combined. As a result of neural summation, the oscillatory haltere input transforms the sustained visual depolarization into a temporally structured train of action potentials that are phase locked to the haltere/wing beat cycle. The strength of the combined visual and haltere inputs is sampled with each stroke of the halteres and represented by the number of action potentials elicited by each haltere-synchronous PSP. In this way the haltere and visual inputs are effectively "spliced" together in a manner similar to that hypothesized to occur in the flight steering system (Heide, 1983; Dickinson, 2005).

An alternative explanation of our results comes from the finding that the haltere steering muscles receive visual inputs, and thus NMNs may receive visual information indirectly via reafferent inputs from the halteres (Chan et al., 1998). This hypothesis is consistent with our extracellular results but cannot alone explain our finding of a subthreshold visual input to the NMNs that is present even in the absence of haltere movements. However, our results do not discount the possibility that NMNs receive indirect visual inputs via the halteres in addition to the direct subthreshold visual input we have identified.

It is probable that the visual responses of type II NMNs are gated not exclusively by the halteres but also by other sensory inputs such as those from the proprioceptive prosternal organ (Preuss and Hengstenberg, 1992; Paulk and Gilbert, 2006), the wind-sensitive antennae, the ocelli (Parsons et al., 2006), or possibly by a central state-dependent signal (Rosner et al., 2009) 
potentially similar to the depolarization seen when the fly spontaneously tried to move during our experiments (supplemental Fig. 1, available at www.jneurosci.org as supplemental material). In addition, a strong-enough visual stimulus might be able to elicit action potentials without nonvisual inputs. However, the well characterized adaptation and gain control mechanisms operating in the LPTCs may attenuate any such effect (for review, see Borst and Haag, 2002).

\section{Phase locking of NMNs}

The visually induced action potentials of all NMNs were found to be phase locked to the haltere oscillation cycle. This is a possible explanation for head oscillations that have been observed at the haltere/wing beating frequency both during free flight (van Hateren and Schilstra, 1999) and in tethered preparations (Rosner et al., 2009). It remains to be seen whether the phase locking has functionally important consequences as in the flight motor system (Heide, 1983; Fayyazuddin and Dickinson, 1996, 1999).

The NMN action potentials remain phase locked to the haltere oscillation even at the high frequencies seen during flight. It appears that this is possible because the haltere-induced PSP sharpens at faster beating frequencies (Fig. 6), preventing the PSPs from completely summating to produce a tonic response. The mechanism underlying the sharpening of the compound PSP is not clear. Possible, nonexclusive, explanations include (i) the addition of an input from an electrically coupled neuron (Fayyazuddin and Dickinson, 1999), (ii) sharpening due to nonlinear conductances (Laurent et al., 1993), or (iii) the individual haltere-driven PSPs arriving more synchronously due to the faster haltere motion.

Different NMN recordings displayed a different baseline response to the haltere stimulus alone, with some neurons not firing at all and some producing up to three phase-locked action potentials per haltere cycle (supplemental Fig. 3, available at www.jneurosci.org as supplemental material). It is not clear whether this difference was due to an intrinsic difference between neurons or due to subtle differences in the haltere stimulus. Sandeman and Markl (1980) used compound action potential recordings to show that the FN NMN population responds most strongly to forward deflections of the ipsilateral haltere. In contrast, our intracellular recordings suggest that FN NMNs can respond to both vertical and horizontal haltere displacements, with each haltere driving a different subset of the NMNs. Such bilateral responses are consistent with the known NMN and haltere neuroanatomy (Sandeman and Markl, 1980; Strausfeld and Seyan, 1985; Chan and Dickinson, 1996; Trimarchi and Murphey, 1997). Our haltere stimulus attempted to replicate the sensory input occurring during straight flight. How the NMNs integrate information from the halteres during rotations of the fly remains to be investigated. The haltere inputs during rotations are likely to also be phase locked to the haltere beating cycle (Pringle, 1948; Nalbach, 1993) and thus may be integrated with the visual inputs in a manner similar to that seen in this study.

\section{Behavioral significance}

Behavioral experiments have shown that blowflies make visually induced gaze-stabilizing head movements only when walking or flying, not when standing still (Hengstenberg, 1991). One possible explanation is that flies may clamp their head to the body when inactive (Gilbert and Bauer, 1998). However, our results are also sufficient to explain this behavioral observation. Blowflies do not beat their halteres when standing, but they do when flying or walking (Sandeman and Markl, 1980). We found that visual inputs affect the action potential responses of type II NMNs, and thus the activity of the innervated neck muscles, only when a nonvisual input such as that from the halteres is present. This may explain why visually induced head movements are seen only during behaviors in which the fly beats its halteres (Hengstenberg, 1991). If this were true, an apparently complex behavioral gating phenomenon (Hengstenberg, 1991) would be explained by a comparatively simple feature of neurons: the action potential threshold nonlinearity.

The gating-like effect we observed may not completely explain the behavioral findings. Tethered flies still make visually induced head movements even when the halteres have been removed (Rosner et al., 2009). This may be due to type I NMNs that respond to visual input even in the absence of haltere input (Milde et al., 1987; Huston and Krapp, 2008). Type I NMNs have smaller extracellular action potential waveforms and higher spontaneous action potential rates (Milde et al., 1987; Huston and Krapp, 2008), features typically associated with smaller-diameter motor neurons that innervate slow muscles. Type II NMNs have the large extracellular waveforms and low spontaneous rates typically associated with larger motor neurons that innervate faster muscles (Henneman et al., 1965; Davis, 1971). Thus, it is possible that haltere movements gate fast, but not slow, visually induced head movements. In agreement with this interpretation, recent behavioral measurements have shown that high-frequency head movements, but not slow ones, are reduced when the halteres are removed (Rosner et al., 2009).

In summary, we have shown that a subpopulation of fly neck motor neurons integrates multisensory inputs in a nonlinear manner. Visual inputs can affect the NMN action potential outputs only if the halteres are simultaneously activated. This gatinglike effect may help explain previous behavioral observations on the context dependence of visually induced head movements. In addition, the results from our intracellular recordings suggest that the action potential threshold is the main cause of the NMN nonlinear integration.

\section{References}

Borst A, Haag J (2002) Neural networks in the cockpit of the fly. J Comp Physiol A Neuroethol Sens Neural Behav Physiol 188:419-437.

Chan WP, Dickinson MH (1996) Position-specific central projections of mechanosensory neurons on the haltere of the blw fly, Calliphora vicina. J Comp Neurol 369:405-418.

Chan WP, Prete F, Dickinson MH (1998) Visual input to the efferent control system of a fly's "gyroscope." Science 280:289-292.

Davis WJ (1971) Functional significance of motorneuron size and soma position in swimmeret system of the lobster. J Neurophysiol 34:274-288.

Dickinson MH (1999) Haltere-mediated equilibrium reflexes of the fruit fly, Drosophila melanogaster. Philos Trans R Soc Lond B Biol Sci 354:903-916.

Dickinson MH (2005) The initiation and control of rapid flight maneuvers in fruit flies. Integr Comp Biol 45:274-281.

Egelhaaf M, Kern R, Krapp HG, Kretzberg J, Kurtz R, Warzecha AK (2002) Neural encoding of behaviourally relevant visual-motion information in the fly. Trends Neurosci 25:96-102.

Elyada YM, Haag J, Borst A (2009) Different receptive fields in axons and dendrites underlie robust coding in motion-sensitive neurons. Nat Neurosci 12:327-332.

Fayyazuddin A, Dickinson MH (1996) Haltere afferents provide direct, electrotonic input to a steering motor neuron in the blowfly, Calliphora. J Neurosci 16:5225-5232.

Fayyazuddin A, Dickinson MH (1999) Convergent mechanosensory input structures the firing phase of a steering motor neuron in the blowfly, Calliphora. J Neurophysiol 82:1916-1926.

Fox JL, Daniel TL (2008) A neural basis for gyroscopic force measurement in the halteres of Holorusia. J Comp Physiol A Neuroethol Sens Neural Behav Physiol 194:887-897. 
Franceschini N (1975) Sampling of the visual environment by the compound eye of the fly: fundamentals and applications. In: Photoreceptor optics (Snyder AW, Menzel R, eds), pp 98-125. New York: Springer.

Gilbert C, Bauer E (1998) Resistance reflex that maintains upright head posture in the flesh fly Neobellieria bullata (Sarcophagidae). J Exp Biol 201:2735-2744.

Gu Y, Angelaki DE, Deangelis GC (2008) Neural correlates of multisensory cue integration in macaque MSTd. Nat Neurosci 11:1201-1210.

Haag J, Wertz A, Borst A (2007) Integration of lobula plate output signals by DNOVS1, an identified premotor descending neuron. J Neurosci 27:1992-2000.

Hateren JH, Schilstra C (1999) Blowfly flight and optic flow II. Head movements during flight. J Exp Biol 202:1491-1500.

Hausen K (1982) Motion sensitive interneurons in the optomotor system of the fly. I. The horizontal cells: structure and signals. Biol Cybern 45:143-156.

Hausen K (1993) Decoding of retinal image flow in insects. In: Visual motion and its role in the stabilization of gaze (Miles FA and Wallman J, ed), pp 203-235. New York: Elsevier.

Heide G (1983) Neural mechanisms of flight control in Diptera. In: Insect flight. BIONA report 2, vol 1 (Nachtigall W, ed), pp 35-52. Stuttgart, Germany: Fischer.

Hengstenberg B (1991) Gaze control in the blowfly Calliphora: a multisensory, two-stage integration process. Semin Neurosci 3:19-29.

Hengstenberg R (1982) Common visual response properties of giant vertical cells in the lobula plate of the blowfly Calliphora. J Comp Physiol A Neuroethol Sens Neural Behav Physiol 149:179-193.

Henneman E, Somjen G, Carpenter DO (1965) Functional significance of cell size in spinal motoneurons. J Neurophysiol 28:560-580.

Huston SJ, Krapp HG (2008) Visuomotor transformation in the fly gaze stabilization system. PLoS Biol 6:e173.

Katz PS (2003) Synaptic gating: the potential to open closed doors. Curr Biol 13:R554-R556.

Krapp HG (2000) Neuronal matched filters for optic flow processing in flying insects. Int Rev Neurobiol 44:93-120.

Laurent G, Seymour-Laurent KJ, Johnson K (1993) Dendritic excitability and a voltage-gated calcium current in locust nonspiking local interneurons. J Neurophysiol 69:1484-1498.

Matheson T, Ditz F (1991) A simple computer-controlled analogue ramp generator for producing multiple ramp-and-hold stimuli. J Neurosci Methods 39:45-52.

Milde JJ, Seyan HS, Strausfeld NJ (1987) The neck motor system of the fly Calliphora erythrocephala. 2. Sensory organization. J Comp Physiol A Neuroethol Sens Neural Behav Physiol 160:225-238.

Nalbach G (1993) The halteres of the blowfly Calliphora I. Kinematics and dynamics. J Comp Physiol A Neuroethol Sens Neural Behav Physiol 173:293-300.

Nalbach G (1994) Extremely non-orthogonal axes in a sense organ for rotation: behavioural analysis of the dipteran haltere system. Neuroscience 61:149-163.

Nalbach G, Hengstenberg R (1994) The halteres of the blowfly Calliphora II. 3-Dimensional organization of compensatory reactions to real and simulated rotations. J Comp Physiol A Neuroethol Sens Neural Behav Physiol 175:695-708.
Parsons MM, Krapp HG, Laughlin SB (2006) A motion-sensitive neurone responds to signals from the two visual systems of the blowfly, the compound eyes and ocelli. J Exp Biol 209:4464-4474.

Paulk A, Gilbert C (2006) Proprioceptive encoding of head position in the black soldier fly, Hermetia illucens (L.) (Stratiomyidae). J Exp Biol 209:3913-3924.

Preuss T, Hengstenberg R (1992) Structure and kinematics of the posternal organs and their influence on head position in the blowfly Calliphora erythrocephala Meig. J Comp Physiol A Neuroethol Sens Neural Behav Physiol 171:483-493.

Pringle JWS (1948) The gyroscopic mechanism of the halteres of Diptera. Philos Trans R Soc Lond B Biol Sci 233:347-385.

Rao Jammalamadaka S, Sengupta A (2001) Topics in circular statistics. Singapore: World Scientific.

Reichert H, Rowell CHF (1986) Neuronal circuits controlling flight in the locust-how sensory information is processed for motor control. Trends Neurosci 9:281-283.

Reichert HR, Rowell CHF, Griss C (1985) Course correction circuitry translates feature detection into behavioural action in locusts. Nature 315:142-144.

Rosner R, Egelhaaf M, Grewe J, Warzecha AK (2009) Variability of blowfly head optomotor responses. J Exp Biol 212:1170-1184.

Sandeman DC (1980) Angular acceleration, compensatory head movements and the halteres of flies (Lucilia serricata). J Comp Physiol 136:361-367.

Sandeman DC, Markl H (1980) Head movements in flies (Calliphora) produced by deflection of the halteres. J Exp Biol 85:43-60.

Sherman A, Dickinson MH (2003) A comparison of visual and halteremediated equilibrium reflexes in the fruit fly Drosophila melanogaster. J Exp Biol 206:295-302.

Sillar KT, Roberts A (1988) A neuronal mechanism for sensory gating during locomotion in a vertebrate. Nature 331:262-265.

Staudacher E, Schildberger K (1998) Gating of sensory responses of descending brain neurones during walking in crickets. J Exp Biol 201: 559-572.

Strausfeld NJ, Gronenberg W (1990) Descending neurons supplying the neck and flight motor of Diptera: organization and neuroanatomical relationships with visual pathways. J Comp Neurol 302:954-972.

Strausfeld NJ, Seyan HS (1985) Convergence of visual, haltere, and prosternal inputs at neck motor neurons of Calliphora erythrocephala. Cell Tissue Res 240:601-615.

Strausfeld NJ, Seyan HS, Milde JJ (1987) The neck motor system of the fly Calliphora erythrocephala. 1. Muscles and motor neurons. J Comp Physiol A Neuroethol Sens Neural Behav Physiol 160:205-224.

Taylor GK, Krapp HG (2007) Sensory systems and flight stability: what do insects measure and why? Adv In Insect Physiol 34:231-316.

Trimarchi JR, Murphey RK (1997) The shaking- $\mathrm{B}^{2}$ mutation disrupts electrical synapses in a flight circuit in adult Drosophila. J Neurosci 17:47004710.

Wertz A, Borst A, Haag J (2008) Nonlinear integration of binocular optic flow by DNOVS2, a descending neuron of the fly. J Neurosci 28:31313140 . 